\title{
Understanding Patient Reluctance to the Remote Monitoring of Cardiac Implantable Electronic Devices
}

\section{Paul Mcloughlin}

University of Ottawa Heart Institute, Ottawa, ON Canada

"Corresponding author: Paul Mcloughlin, University of Ottawa Heart Institute, Ottawa, ON Canada,Tel: 606- 16137693742; E-mail: pmcloughlin@ottawaheart.ca Received date: Apr 20, 2018; Accepted date: Jun 28, 2018; Published date: July 10, 2018

Copyright: (c) 2018 Mcloughlin P. This is an open-access article distributed under the terms of the Creative Commons Attribution License, which permits unrestricted use, distribution, and reproduction in any medium, provided the original author and source are credited.

\begin{abstract}
Objective: To understand patient's reasoning for declining remote monitoring of their cardiac device. Is a patient more likely to proceed with remote monitoring if they are aware of the benefits and limitations of remote monitoring?

Background: It is now possible to assess pacemakers and defibrillators remotely through the use of personal monitors. Remote monitoring has many evidence based benefits for the patient and device clinic, and is now an integral part of the standard of medical care for CIED patients in Canada. Despite this, a minority of patients decline remote monitoring. We have a poor understanding as to why this might be, with little research in contemporary literature looking at this area.
\end{abstract}

Methods: A descriptive survey, questionnaire study was used having both quantitative and qualitative features. This allowed for the primary reasons for patients declining remote monitoring to be concisely identified, using thematic analysis. It assessed a patient's knowledge of the benefits of remote monitoring, looking for a relationship between this and likelihood to proceed with remote monitoring.

Results: Loss of human contact appears to be a predominant concern, confirming patient's reported experiences in Ottenberg's study and as suggested in the 2015 HRS statement. Privacy and security fears were also highlighted. Surprisingly fear of technology, in a mainly elderly population, was rarely mentioned. Half of the participants in this study stated that they would be likely to proceed with remote monitoring after having read the evidence based benefits for its use.

Conclusions and Recommendations: Patient acceptance of remote monitoring can be improved by educating them to its benefits and limitations. To alleviate privacy concerns, device companies should look at the feasibility of having their remote monitoring servers physically based in Canada.

Abbreviations CIED-Cardiac Implantable Electronic Device; CHRSCanadian Heart Rhythm Society; HRS-Heart Rhythm Society; ICDImplantable Cardioverter Defibrillator; RM-Remote Monitoring; USFDA-United States Food and Drug Administration

Keywords: Remote monitoring; Cardiac devices; Pacemakers; ICDs; Telemedicine; Internal cardioverter defibrillator

\section{Introduction}

The first pacemaker was implanted in 1958 and since then the use of Cardiac Implantable Electronic Devices (CIEDs) has grown rapidly. It is now possible to assess pacemakers and defibrillators remotely using personal monitors. This information is sent to a secure server to which the patient's device clinic has access via a standard web browser. Remote monitoring has many benefits for the patient and device clinic and is now the standard of care for CIED patients in the United States and an integral part of the standard of medical care for CIED patients in Canada [1-3].

Despite the evidence based benefits to the patient, and the recommendation of remote monitoring by the national Heart Rhythm Societies, a minority of patients decline remote monitoring. We have a poor understanding as to why this might be, with little research in contemporary literature looking at this area. Freeman clearly articulates the need for research as to why patients are declining remote monitoring ... "Additionally, research must be conducted to better understand patient ... impediments to adopting remote monitoring and methods to address these barriers'[4].

A mixed methods questionnaire was designed to reveal, in their own words, why patients had previously declined monitoring. It was also designed to assess patient's knowledge of the benefits of remote monitoring. Patient's concerns and knowledge gaps could then be addressed through consistent, evidence informed, on-going education, commencing before device implant.

\section{History of remote monitoring}

Remotely obtaining information from a patient's cardiac device is not a new concept in pacemaker follow-up. In 1971, trans-telephonic monitoring (TTM) was introduced to supplement in clinic visits [5]. The monitor is connected to an analogue phone-line and electrodes are placed on the patient. An ECG tracing is sent, with a magnet applied, and then not applied, to the pacemaker. The information gleaned from TTM is limited. 
The birth of modern digital remote monitoring was 2001 when Biotronik released their Home Monitoring system in Europe. In 2002, in the United States, Medtronic released their Carelink monitoring system. All five major cardiac device companies now have a remote monitoring system and remote capable pacemakers and ICDs. It was now possible to interrogate a pacemaker or ICD and gather all the same information that would usually have required an in-clinic visit to obtain.

However, true remote patient monitoring came with the advent of wireless ICDs and pacemakers. Intermittent remote interrogations were now replaced by true remote monitoring of devices. Once set-up these monitors required no physical interaction with the patient. The monitor is typically placed within $4 \mathrm{~m}$ of where the patient sleeps, with transmissions usually sent during the night. The monitor will work anywhere in the world that has mains electricity and cellular or Wi-Fi connectivity. The collected information is sent encrypted to servers maintained by the device company. The patient's device clinic has access to this information via a standard web browser.

\section{The evidence for the benefits of remote monitoring}

Over time the benefits of remote monitoring to both the patient and the device clinic became apparent. Remote monitoring is a solution to the problem of clients who live long distances from the specialised device clinic. Historically this has been an issue for Canadian centres, with some patients in Arctic Canada living over two thousand Kilometres from their device clinic. However, the benefits to remote monitoring apply to all patients irrespective of their distance from the device clinic. Numerous prospective, retrospective, and randomized clinical trials showed positive patient outcomes. Remote monitoring has many benefits for the patient including reduced clinic visits, early warning of battery, device, or lead problems, and earlier notification of dangerous heart rhythms or other changes in cardiac status [6-10]. A reduction in inappropriate shocks has also been shown for patients on remote monitoring [11]. Some studies have shown a mortality benefit to patients on remote monitoring $[12,13]$ though the evidence is not conclusive [14]. Improved clinic efficiency, reduced cost to patient and health care system, and improved quality of life were also shown [9,15-17] (Figure 1).

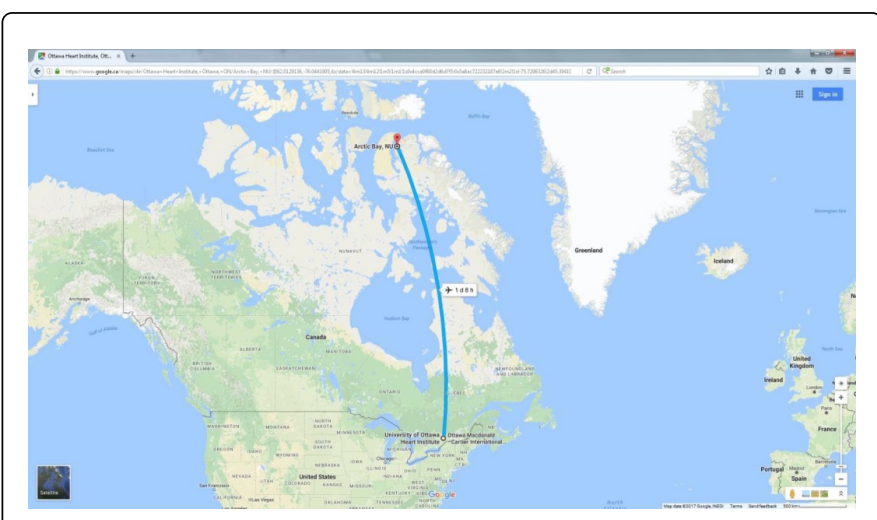

Figure 1: Distance travelled by some patients for a device check.

\section{Methods}

From the literature review, no prior research was identified specifically looking at patients who had previously declined remote monitoring. Due to the number of patients identified and the initial nature of the primary question, a descriptive survey, questionnaire study was chosen. As the question and hypothesis were very narrow and focused, a previously used and validated questionnaire was not available. The questionnaire has both quantitative and qualitative features and allowed for the primary reasons why patients are declining remote monitoring to be concisely identified [18]. A format with statements using tick box categories was chosen. The advantages to this format are a questionnaire that is easy to understand and relatively quick to complete. It also generates data suitable for nonparametric statistical analysis and display of descriptive statistics [19]. It assessed patient's knowledge of the benefits of remote monitoring, looking for an association between knowledge and a patient's likelihood of accepting.

To answer the question as to why some patients decline remote monitoring, it was first necessary to identify the patients who had previously declined at an academic Heart Institute in Southern Canada. The nursing clinic notes, on the Paceart clinic database, of patients with newly implanted Medtronic devices from April 2015October 2016 were manually checked. From this, 45 patients were identified with a documented decline of remote monitoring. It is likely some patients declined and it was not documented. However, the sample is as comprehensive as could be reasonably obtained. Of these 45 patients, 13 had previously stated, as documented on the hospital electronic health record system, that they did not wish to be contacted for research purposes (Figure 2).

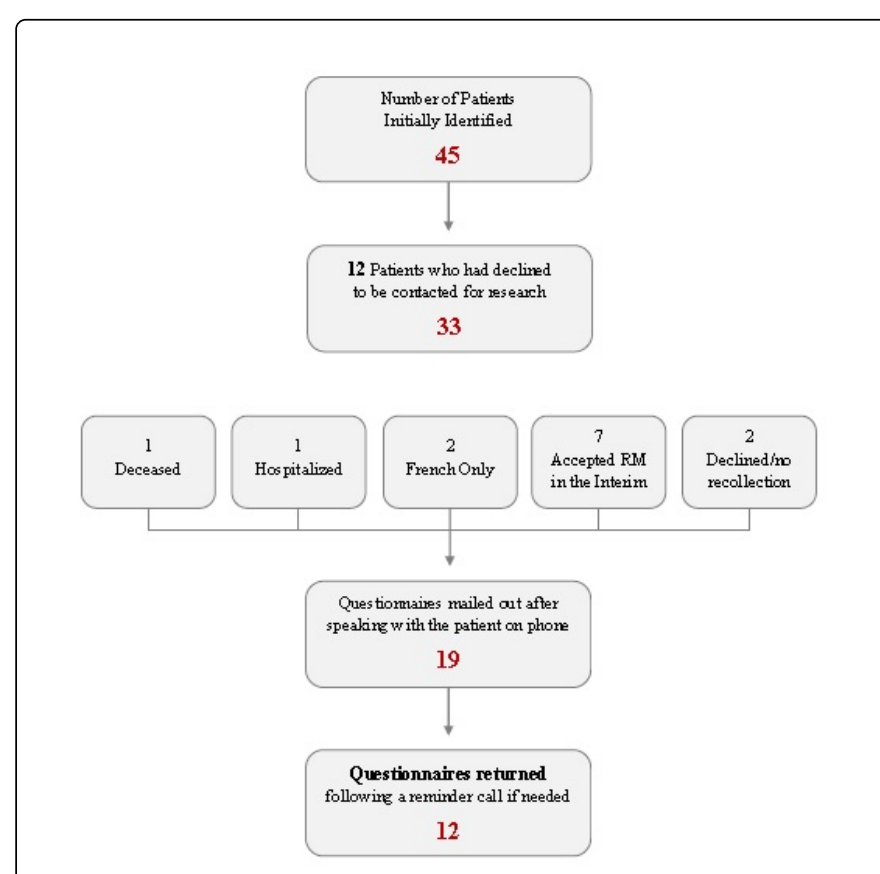

Figure 2: From identification of patients to return of questionnaires-The realities of research.

\section{Results}

A total of 19 questionnaires were mailed out. The patients were called before and after mailing, and twelve questionnaires were returned, one of which was returned blank. Of those questionnaires completed nine of the participants were male and two female. The 
Page 3 of 7

youngest participant was 58 years old with most respondents more than 70 years old.

The questionnaires returned offer some enlightening insights into patient's reasoning for declining remote monitoring. In their own words participants shed some light on the main focus of this study, namely understanding patient's reluctance to remote monitoring. These themes will be grouped under headings consistent with the three possible reasons for declining remote monitoring, namely technology, privacy concerns, and loss of human contact, as posited by the HRS3, and Ottenberg's 2 themes of perspectives and perceptions of usefulness.

\section{Part one}

"What was your main reason, or reasons, for declining remote monitoring?"Technology:

One participant wrote ..."fear of the unknown (technology)", an area previously highlighted in the HRS3 position statement on remote monitoring. Many device patients are elderly and may have greater challenges with newer technology. It must be said that the device companies have done a great job in making the monitors as simple as possible. Following a 2-step process taking two minutes, no further patient interaction is required. It will be important to let the patient know that the device company, and their device clinic, will help them throughout the set-up process.

Another Participant, who described themselves as ..."a very nervous person", wrote of their belief that the monitor would increase their level of stress. They described not being technically inclined, and not needing the perceived burden of stress. One participant wrote of feeling uncomfortable with any new device such as a remote monitor because they ... "do not have a hand-held device or a cell phone".

\section{Perspectives and perceptions of usefulness}

"We do not see things as they are. We see things as we are" [20].

"I do not want to be constantly reminded that I have an implanted device, particularly since it is not necessary for normal daily living," is how one participant describes their perception of the usefulness of a remote monitor. This same participant also spoke of not wanting another piece of equipment in their bedroom taking up space.

Often what we think a patient hears, and what the patient actually hears, are two very different things. Internet access is not required for cellular remote monitoring and is not mentioned in-clinic as a prerequisite for monitoring. Despite this a participant writes that ... "I had no access to the internet and I was told I would need access. My son has moved back home, he has a computer so I could now participate". Another Participant wrote "not sure if I understand what remote monitoring means, if it will affect me in any way." A further participant wrote that they did not remember declining remote monitoring. One participant called the clinic after receiving the questionnaire to report having no recollection of the study or declining remote monitoring. This was after the study had being explained fully during a telephone conversation. The questionnaire was returned uncompleted with only ..."I do not remember the operation. I do not feel the implant at all. I feel great and do not know it is there." It is difficult to read these replies without feeling a sense of sadness at the discontinuity of what we say as health care professionals and what our patients hear and perceive.

\section{Loss of human contact}

One participant succinctly highlighted loss of human contact as a concern, writing of a ... "desire to be seen/checked by real competent doctors/nurses to whom I can address/questions/concerns." Another participant wrote that they liked being seen by a cardiologist every 6 months. One participant articulates their feelings quite clearly ... "I enjoy the one on one with the staff at the defibrillator clinic. Any of my questions or concerns are always answered and totally explained. I was a little concerned about losing this." Along the same lines another participant writes ... "I still prefer physically attending and having personal contact." Similarly, another participant wrote ..."I prefer one on one anyway."

Loss of human contact was a predominant theme for patient's reasons for declining remote monitoring. Another participant perceived they would lose the ..." ability to travel to the Heart Institute for monitoring; ability to discuss any issues with appropriate personal on a one to one, face to face basis." They saw the monitor as impersonal, and they would miss the ability to interact with appropriate personnel ..."remote monitoring, while advantageous, lacks the personal interaction which is beneficial to the patient."

It is important to reassure the patient that they will still attend clinic at least annually, and remote monitoring complements, not replaces usual care. As highlighted in Petersen's study [21], with $84 \%$ of patients requesting more information after a routine transmission, it is vital to engage and inform the patient in their remote monitoring follow-up.

In a busy device clinic, we can sometimes forget the importance of giving the patient time and space to comprehend and digest the information they are given. This was emphasized by one participant who wrote ..." the questions were asked ... "at such speed that I had difficulty in total comprehension. Therefore, I choose the safety of continuing to deal with real people".

\section{Privacy and security}

For those caring for patients with CIEDs, issues surrounding the security and privacy of patient's medical information is currently at the forefront. In regards to privacy, there is a concern with remote monitoring and the collection of personal medical information, that this information is no longer located at the point of care [1].

As to why they declined remote monitoring one participant wrote ..."I object to having my personal medical information stored on a server in the USA." In the further comments section the same participant wrote ..."Also, we are now in the era of President Donald Trump?'

Regarding the security of remote monitoring one participant simply wrote ..."I am declining remote monitoring for security reasons." Unfortunately, the participant did not expand on their reasoning. This highlights the possible inferiority of questionnaires, as compared to indepth interviews, for obtaining more detailed information on certain topics. Question seven informs that the device information is transmitted encrypted and stored safely on secure servers. Despite this a participant wrote ..."Encrypted information is hacked into on a regular basis."

\section{Part Two}

Questions 1-8 are evidence informed statements that summarize some of what we know at present about remote monitoring. The 
Citation: Mcloughlin P (2018) Understanding Patient Reluctance to the Remote Monitoring of Cardiac Implantable Electronic Devices . J Nurs

Page 4 of 7

questions were designed to assess a patient's knowledge of remote monitoring and to see if this was associated with an intention to proceed with remote monitoring (Question 9). Two participants did not complete part two and one participant partially completed. Nine participants answered all of questions 1-8 in part two (Figure 3-11).

This research suggests that the three main reasons given by patients for declining remote monitoring are loss of human contact, privacy and security concerns, and fear of technology. Loss of human contact appears to be a predominant concern, confirming patient's reported experiences in Ottenberg's study [2]. Surprisingly fear of technology, in a mainly elderly population, was rarely mentioned. This study also highlighted that there is much room for improvement when informing patients of the benefits of remote monitoring. Half of the participants in this study stated that they would be likely to accept remote monitoring after having read the evidence based benefits for its use.

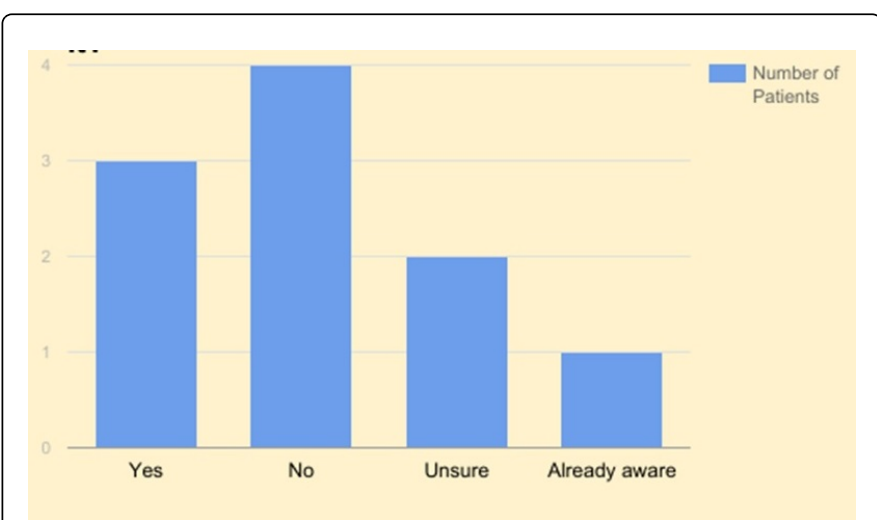

Figure 3: Would knowing RM is the standard of care make it more likely you would choose it?

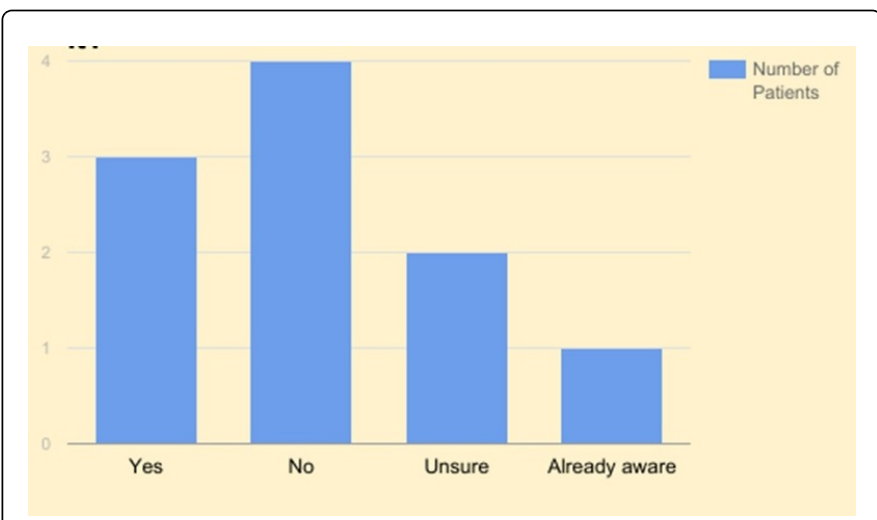

Figure 4: $98 \%$ of patients stay on RM. Would knowing this make it more likely you would choose RM?

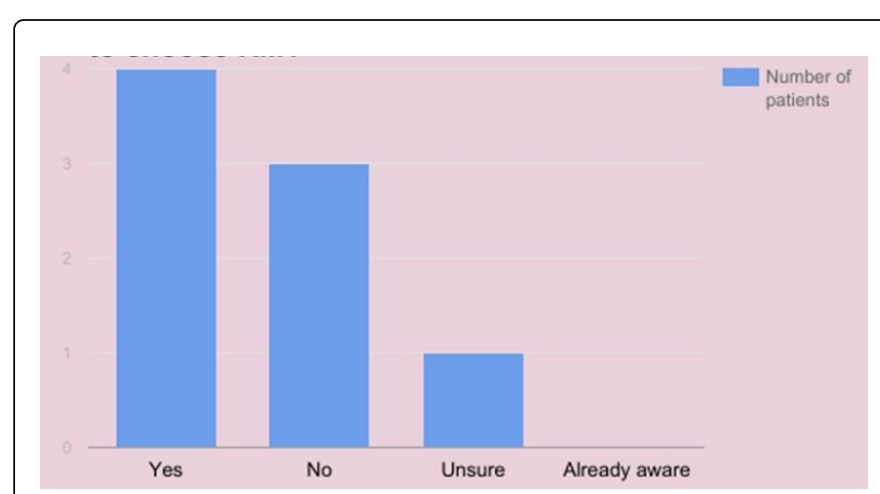

Figure 5: RM may reduce inappropriate shocks. Would knowing this make you more likely to choose RM?

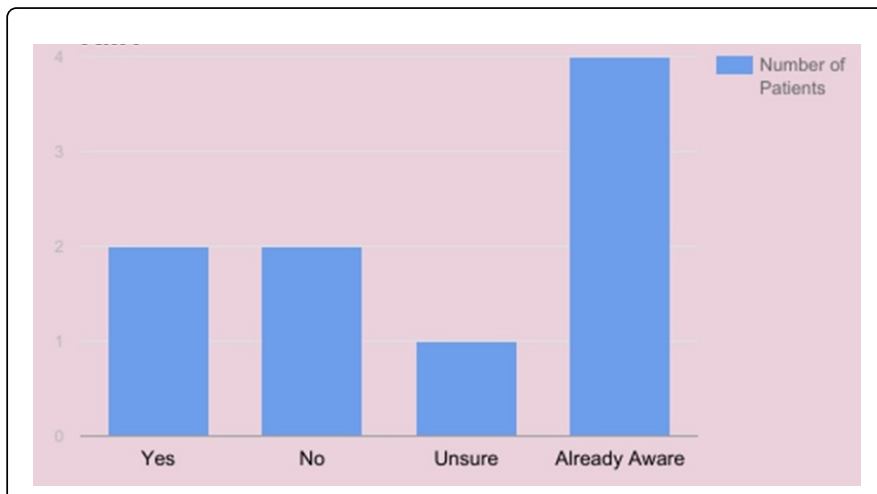

Figure 6: There is no cost for RM. Would knowing this make it more likely you would choose RM?

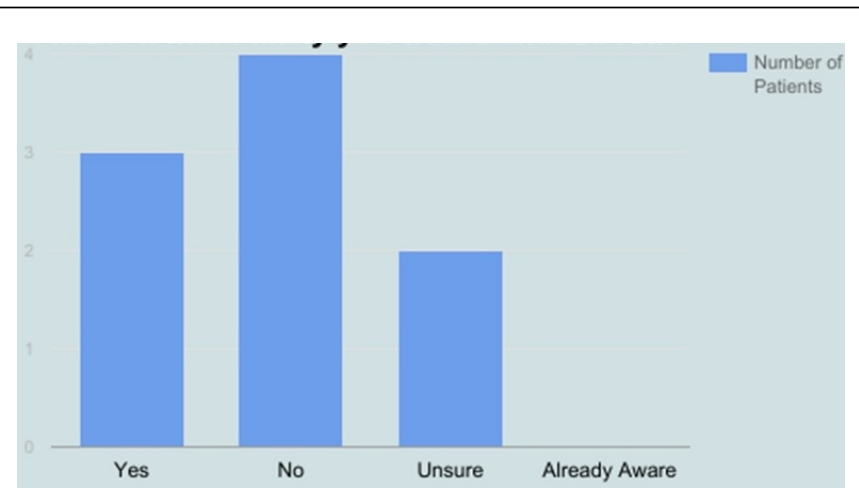

Figure 7: RM requires no computer experience and takes 2 mins to set-up. Would knowing this make it more likely you would choose RM? 


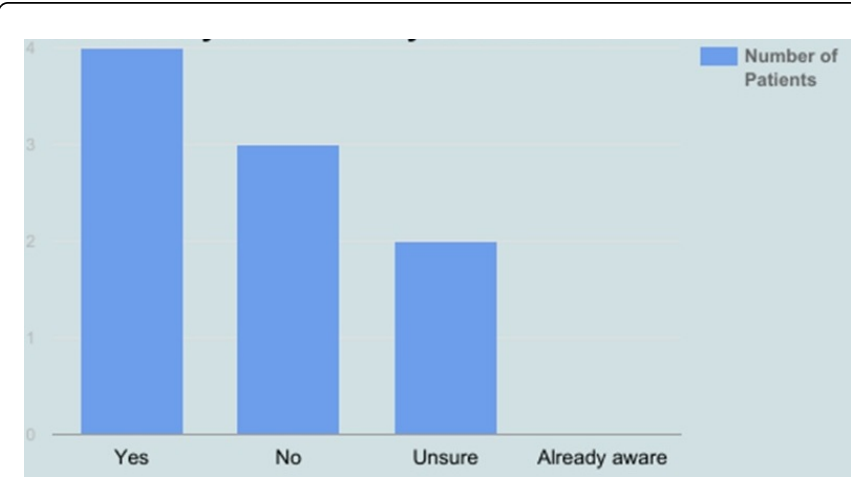

Figure 8: RM gives early warning of low battery, device issues and lead problems. Would this make you more likely to choose RM?

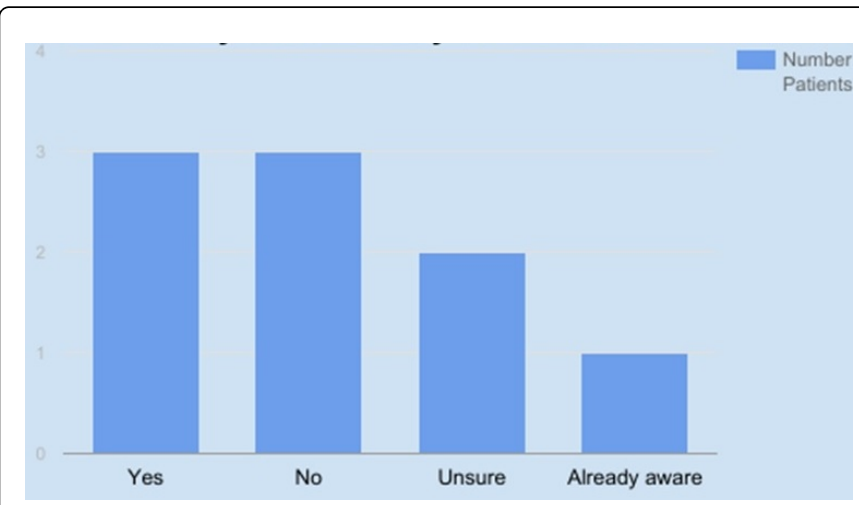

Figure 9: Your data is sent encrypted and securely and may only be read by the clinic. Would this make you more likely to choose RM?

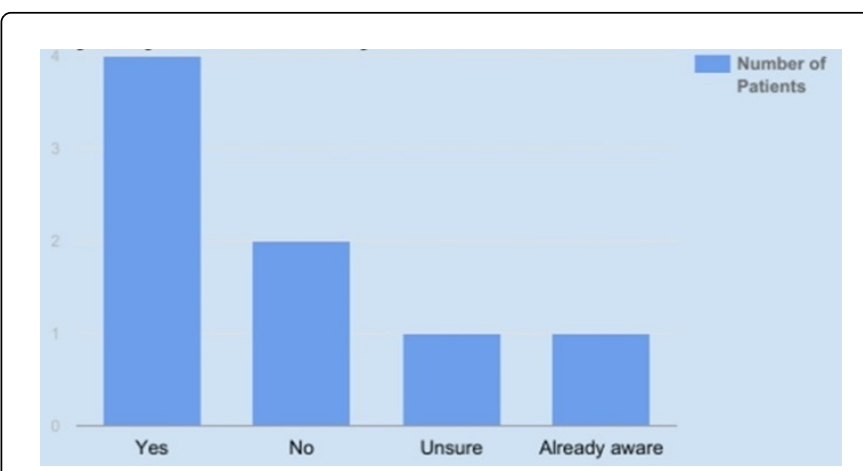

Figure 10: RM complements not replaces usual care. Patients must still be seen at least yearly in clinic. Likely to choose RM?

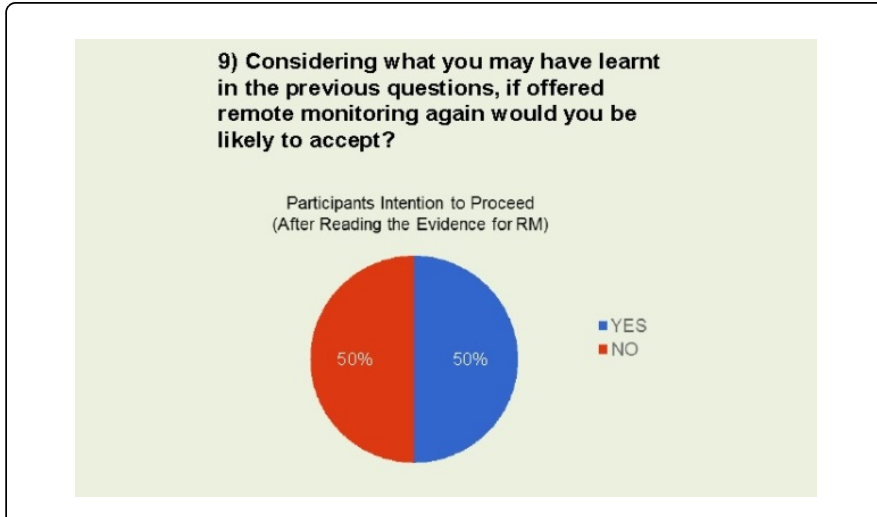

Figure 11: Considering what you may have learnt in the previous questions, if offered remote monitoring again would you be likely to accept?

\section{Limitations}

This study has several limitations. Due to time constraints French only speaking patients were not enrolled. Also, no First Nation patients from Northern Canada were identified in the initial patient search. It is unclear as to why this is. Experience has shown that even when a patient in Arctic Canada has cellular connectivity other issues frequently result in the discontinuation of remote monitoring. Understanding the perspectives and experience of Northern patients, and how to best help them receive person centred and up to date care for their cardiac devices, is an area for further study.

Readers should interpret the findings with caution due to the small number of completed questionnaire. Nevertheless, this research provides a descriptive account, in their own words, as to why patients may decline remote monitoring.

\section{Discussion}

\section{Loss of human contact}

"Remote monitoring, while advantageous, lacks the personal interaction which is beneficial to the patient." This was one answer given by a participant for declining remote monitoring. This personal touch is also beneficial to the nurse and vital for therapeutic care. A concern is, as remote monitoring becomes a bigger aspect of the work day and one begins to lose the interaction with the patient in-clinic, will one still be nursing?

It is important to remind the patient that even when on remote monitoring they continue to be closely followed by their device clinic and must be seen in person at least yearly. It is likely that a change to remote monitoring is more difficult for patients who have become familiar with being seen in clinic every 6 mon. It is recommended that the concept of remote monitoring is introduced by the physician at the patient's initial pre-implant visit. The physician could briefly mention the benefits and limitations, and state that remote monitoring is the standard of care and recommended by the CHRS. The patient will then be started on remote monitoring at their first post-implant visit. A combination of in-clinic and remote monitoring then becomes the norm for the patient. 


\section{Privacy and security}

A participant wrote of their objection to having their personal medical information stored on servers in the USA. In 2012, the Medical Advisory Secretariat (MAS) of Ontario published an evidence based, systematic review of the cost-effectiveness and safety of remote monitoring of cardiac devices [22]. The MAS review highlighted concerns regarding the upkeep of the infrastructure and the fact that the systems were managed by the device manufacturers. Historically, any information concerning a patient and their device would remain at the hospital at which the patient was implanted and followed. However, with remote monitoring the information is now held at distance from the device clinic. Sometimes, as is the case in Canada, this patient medical information may be stored in a different country, such as the USA. It is technically possible that due to the US Patriot Act, a Canadian's health information, stored on servers in the USA could, without the client's consent, be viewed by people outside of their circle of care. Concern regarding the storage of sensitive information outside Canada is not unique to remote monitoring. Google Inc. has recently announced their first Canadian "cloud region" in Montreal [23]. This will allow sensitive data to stay within Canada, allowing organizations that deal with sensitive data or are heavily regulated, such as financial institutions or the health care sector, to stay clear of the US Patriot Act.

Worldwide hundreds of thousands of people are now remotely monitored. There have been millions of remote transmissions sent and received. At present, there is no recorded case, in the medical literature, of a patient's information having been compromised in this way. This is an extremely unlikely scenario, but one nevertheless, that the client needs to be aware of for informed consent to take place. Again, it must be stressed that only a tiny part of a patient's medical information is transmitted and stored remotely, namely minimal demographics and their cardiac device programming and functioning. At present the benefits of remote monitoring far outweigh the very minimal privacy risks associated with having the information stored outside the hospital's jurisdiction [24].

One participant wrote that they declined remote monitoring for security reasons. Though only briefly mentioned in the literature, the security of radio-frequency enabled cardiac devices and their susceptibility to "hacking" is a topic becoming more widely debated. In January 2017, the USFDA issued a safety communication concerning St Jude cardiac devices and cyber security vulnerabilities [24]. It stated the USFDA had reviewed information regarding potential cyber security vulnerabilities with St. Jude Medical's Merlin@home transmitter. A firmware fix is now available. However this fix may cause more harm than, what is a present, only a potential problem [25]. These are potential issues and at no time have a patient suffered ill or had their device compromised. The USFDA has determined that the health benefits to patients from continued use of remote monitoring outweigh the risks. Patients are reminded to keep their remote monitors plugged in at all times so they can receive the necessary software updates and patches. It is important however, to remain aware that any medical device connected to a communications network may have cyber security vulnerabilities that could be exploited by unauthorised users.

\section{Conclusion}

Pacemakers and internal defibrillators save lives and reduce suffering. These devices can now be assessed remotely. Remote monitoring has benefits for the patient, device clinic, and health care system. However, no intervention is without possible negative consequences. This study sought to understand why some patients decline a largely beneficial treatment. It was thought that educating patients to the evidence based benefits of remote monitoring may increase their likelihood of proceeding with remote monitoring.

In their own words participants shared their concerns, perceptions, and fears regarding remote monitoring. Loss of human contact was a primary concern. Issues concerning the security of remote monitoring and potential loss of privacy were highlighted. The technological aspect of remote monitoring negatively affected a minority of patients.

The secondary objective of the study, was to assess patient's knowledge of the benefits of monitoring, and if this may be related to acceptance. From the answers, it was clear that patients were not aware of the evidence based benefits of remote monitoring. Half of the patients, after reading of the benefits, would now be likely to proceed with remote monitoring.

\section{Recommendations}

The following evidence informed recommendations are made:

-Patients are to be briefly introduced to the concept of remote monitoring, by their physician, at the pre-implant visit if possible. The seed is planted.

-A clear and concise information and FAQ sheet explaining the benefits and limitations of remote monitoring is to be included with the device guide for patients.

-At the first post implant visit consent for remote monitoring is obtained following an overview of the limitations and evidence based benefits. Patients are informed they will be followed by a combination of in clinic and remote visits. As this will be their norm a perceived loss of human contact is less likely to be felt.

-The device companies are to be encouraged to consider the feasibility of having their remote monitoring servers physically based in Canada.

\section{References}

1. Yee R, Verma A, Beardsall M, Fraser J, Philippon F, et al. (2013) Canadian Cardiovascular Society/Canadian Heart Rhythm Society joint position statement on the use of remote monitoring for cardiovascular implantable electronic device follow-up. Can J Cardiol 29: 644-651.

2. Ottenberg AL, Swetz KM, Mueller LA, Gerhardson S, Mueller PS (2013) We as Human Beings Get Farther and Farther Apart: The experiences of patients with remote monitoring systems. Heart Lung 42: 313-319.

3. Slotwiner D, Varma N, Akar JG, Annas G, Beardsall M, et al. (2015) HRS Expert Consensus Statement on remote interrogation and monitoring for cardiovascular implantable electronic devices. Heart Rhythm 12: 69-100.

4. Freeman JV, Saxon L (2015) Remote monitoring and outcomes in pacemaker and defibrillator patients: Big data saving lives? J Am Coll Cardiol 65: 2611-2613.

5. Movsowitz C, Mittal S (2011) Remote patient management using implantable devices. J Interv Card Electrophysiol 31: 81-90.

6. Akar JG, Bao H, Jones PW, Wang Y, Varosy PD, et al. (2015) Use of remote monitoring is associated with lower risk of adverse outcomes among patients with implanted cardiac defibrillators. Circ Arrhythm Electrophysiol 8: 1173-1180.

7. Crossley GH, Boyle A, Vitense H, Chang Y, Mead RH, et al. (2011) The CONNECT (Clinical Evaluation of Remote Notification to Reduce Time 
Citation: Mcloughlin P (2018) Understanding Patient Reluctance to the Remote Monitoring of Cardiac Implantable Electronic Devices . J Nurs Care 7: 460. doi:10.4172/2167-1168.1000460

Page 7 of 7

to Clinical Decision) trial the value of wireless remote monitoring with automatic clinician alerts. J Am Coll Cardiol 57: 1181-1189.

8. Guédon-Moreau L, Lacroix D, Sadoul N, Clémenty J, Kouakam C, et al. (2013) A randomized study of remote follow-up of implantable cardioverter defibrillators: Safety and efficacy report of the ECOST trial. Eur Heart J 34: 605-614

9. Landolina M, Perego GB, Lunati M, Curnis A, Guenzati G, et al. (2012) Remote monitoring reduces healthcare use and improves quality of care in heart failure patients with implantable defibrillators: the evolution of management strategies of heart failure patients with implantable defibrillators (EVOLVO) study. Circulation 125: 2985-2992.

10. Varma N, Epstein AE, Irimpen A, Schweikert R, Love C, et al. (2010) Efficacy and safety of automatic remote monitoring for implantable cardioverter-defibrillator follow-up: The Lumos-T safely reduces routine office device followup (TRUST) trial. Circulation 122: 325-332.

11. Guédon-Moreau L, Kouakam C, Klug D, Marquié C, Brigadeau F, et al. (2014) Decreased delivery of inappropriate shocks achieved by remote monitoring of ICD: A sub study of the ECOST trial. J Cardiovasc Electrophysiol 25: 763-770.

12. Hindricks G, Taborsky M, Glikson M, Heinrich U, Schumacher B,et al. (2014) Implant-based multiparameter telemonitoring of patients with heart failure (IN-TIME): A randomised controlled trial. Lancet 384: 583-590.

13. Saxon LA, Hayes DL, Gilliam FR, Heidenreich PA, Day J, et al. (2010 )Long-term outcome after ICD and CRT implantation and influence of remote device follow-up: the ALTITUDE survival study. Circulation 122: 2359-2367.

14. Parthiban N, Esterman A, Mahajan R, Twomey DJ, Pathak RK, et al. (2015) Remote monitoring of implantable cardioverter-defibrillators: A systematic review and meta-analysis of clinical outcomes. Journal of the American College of Cardiology 65: 2591-2600.

15. Calò L, Gargaro A, De Ruvo E, Palozzi G, Sciarra L, et al. (2013) Economic impact of remote monitoring on ordinary follow-up of implantable cardioverter defibrillators as compared with conventional inhospital visits. A singlecenter prospective and randomized study. J Interv Card Electrophysiol 37: 69-78.

16. Hindricks G, Elsner C, Piorkowski C, Taborsky M, Geller JC, et al. (2013) Quarterly vs. yearly clinical follow-up of remotely monitored recipients of prophylactic implantable cardioverter-defibrillators: Results of the REFORM trial. Eur Heart J 35: 98-105.

17. Ricci RP, Vicentini A, D'Onofrio A, Sagone A, Rovaris G, et al. (2017) Economic analysis of remote monitoring of cardiac implantable electronic devices: Results of the health economics evaluation registry for remote follow-up (TARIFF) study. Heart Rhythm 14: 50-57.

18. Bell J (2010) Doing your research project: a guide for first-time researchers in education, health and social science. McGraw-Hill Open University Press, Maidenhead, Berkshire, New York.

19. Boynton PM, Greenhalgh T (2004) Selecting, designing, and developing your questionnaire. BMJ 328: 1312-1315.

20. Covey SR (2004) The 7 habits of highly effective people: Restoring the character ethic. New York.

21. Petersen HH, Larsen MC, Nielsen OW, Kensing F, Svendsen JH, et al. (2012) Patient satisfaction and suggestions for improvement of remote ICD monitoring. J Interv Card Electrophysiol 34: 317-324.

22. Pron G, Ieraci L, Kaulback K (2012) Internet-based device-assisted remote monitoring of cardiovascular implantable electronic devices: An evidence-based analysis. Ont Health Technol Assess Ser 12: 1-86.

23. Connell J (2017) Google inc. announces first canadian 'cloud region' in montreal, allows sensitive data to stay within borders.

24. USFDA (2017) Cybersecurity vulnerabilities identified in St. Jude medical's implantable cardiac devices and Merlin@home transmitter: FDA safety communication, USA.

25. Baranchuk A (2018) Cybersecurity for cardiac implantable electronic devices: What should you know? J Am Coll Cardiol 71: 1284-1288. 